Article

\title{
Functionalization of 3D Chitinous Skeletal Scaffolds of Sponge Origin Using Silver Nanoparticles and Their Antibacterial Properties
}

\author{
Tomasz Machałowski 1,2(D), Maria Czajka ${ }^{3}\left(\mathbb{D}\right.$, Iaroslav Petrenko ${ }^{2}$, Heike Meissner ${ }^{4}$, \\ Christian Schimpf ${ }^{5}$, David Rafaja ${ }^{5}$, Jerzy Ziętek ${ }^{6}$, Beata Dzięgiel ${ }^{6}$, tukasz Adaszek ${ }^{6}$, \\ Alona Voronkina ${ }^{7}$ (D), Valentin Kovalchuk ${ }^{8}$, Jakub Jaroszewicz ${ }^{9}$, Andriy Fursov ${ }^{2}$, \\ Mehdi Rahimi-Nasrabadi 10,11, Dawid Stawski ${ }^{3} \mathbb{D}$, Nicole Bechmann ${ }^{12,13,14,15}{ }^{(D)}$, \\ Teofil Jesionowski ${ }^{1, *}$ (D) and Hermann Ehrlich ${ }^{2,16, *}$
}

1 Institute of Chemical Technology and Engineering, Faculty of Chemical Technology, Poznan University of Technology, Berdychowo 4, 60965 Poznan, Poland; tomasz.g.machalowski@doctorate.put.poznan.pl

2 Institute of Electronics and Sensor Materials, TU Bergakademie Freiberg, Gustav-Zeuner str. 3, 09599 Freiberg, Germany; iaroslavpetrenko@gmail.com (I.P.); andriyfur@gmail.com (A.F.)

3 Institute of Material Science of Textiles and Polymer Composites, Lodz University of Technology, Zeromskiego 16, 90924 Lodz, Poland; maria.czajka@dokt.p.lodz.pl (M.C.); dawid.stawski@p.lodz.pl (D.S.)

4 Department of Prosthetic Dentistry, Faculty of Medicine and University Hospital Carl Gustav Carus of Technische Universität Dresden, Fetscherstraße 74, 01307 Dresden, Germany; heike.meissner@uniklinikum-dresden.de

5 Institute of Materials Science, TU Bergakademie Freiberg, Gustav-Zeuner str. 5, 09599 Freiberg, Germany; schimpf@iww.tu-freiberg.de (C.S.); rafaja@ww.tu-freiberg.de (D.R.)

6 Department of Epizootiology and Clinic of Infectious Diseases, Faculty of Veterinary Medicine, University of Life Sciences, Akademicka 13, 20612 Lublin, Poland; achantina@op.pl (J.Z.); beatadziegiel@o2.pl (B.D.); lukaszek0@wp.pl (Ł.A.)

7 Department of Pharmacy, National Pirogov Memorial Medical University, Pirogov str. 56, 21018 Vinnitsa, Ukraine; algol2808@gmail.com

8 Department of Microbiology, National Pirogov Memorial Medical University, Pirogov str. 56, 21018 Vinnitsa, Ukraine; valentinkovalchuk2015@gmail.com

9 Materials Design Division, Faculty of Materials Science and Engineering, Warsaw University of Technology, Woloska 141, 02507 Warsaw, Poland; jakubjaroszewicz@wp.pl

10 Chemical Injuries Research Center, Systems Biology and Poisonings Institute, Baqiyatallah University of Medical Sciences, Tehran 1951683759, Iran; rahiminasrabadi@gmail.com

11 Faculty of Pharmacy, Baqiyatallah University of Medical Sciences, Tehran 1951683759, Iran

12 Institute of Clinical Chemistry and Laboratory Medicine, University Hospital Carl Gustav Carus, Technische Universität Dresden, Fetscherstrasse 74, 01307 Dresden, Germany; nicole.bechmann@uniklinikum-dresden.de

13 Department of Medicine III, University Hospital Carl Gustav Carus, Technische Universität Dresden, Fetscherstrasse 74, 01307 Dresden, Germany

14 Department of Experimental Diabetology, German Institute of Human Nutrition Potsdam-Rehbruecke, Arthur-Scheunert-Allee 114, 14558 Nuthetal, Germany

15 German Center for Diabetes Research (DZD), Ingolstaedter Landstrasse 1, 85764 München-Neuherberg, Germany

16 Center for Advanced Technology, Adam Mickiewicz University, Uniwersytetu Poznańskiego 10, 61614 Poznan, Poland

* Correspondence: Teofil.Jesionowski@put.poznan.pl (T.J.); Hermann.Ehrlich@esm.tu-freiberg.de (H.E.)

Received: 14 May 2020; Accepted: 8 June 2020; Published: 10 June 2020

Abstract: Chitin, as one of nature's most abundant structural polysaccharides, possesses worldwide, high industrial potential and a functionality that is topically pertinent. Nowadays, the metallization of naturally predesigned, 3D chitinous scaffolds originating from marine sponges is drawing focused attention. These invertebrates represent a unique, renewable source of specialized chitin due to 
their ability to grow under marine farming conditions. In this study, the development of composite material in the form of 3D chitin-based skeletal scaffolds covered with silver nanoparticles (AgNPs) and Ag-bromide is described for the first time. Additionally, the antibacterial properties of the obtained materials and their possible applications as a water filtration system are also investigated.

Keywords: chitin; sponges; 3D scaffolds; AgNPs; antibacterial properties; Aplysina aerophoba

\section{Introduction}

More than 1.2 billion people have no access to clean drinking water [1]. Additionally, 1.8 billion people drinking water from sources susceptible to fecal contamination results in the death of approx. one million children every year [2]. Contaminated potable water, with pathogenic microorganisms (i.e., Escherichia coli infection) represents one of the world's most serious health threats [3]. Consequently, the development of new materials, which are capable of mitigating the risk of bacterial contamination in water needs to be an ongoing, crucial area of research [4].

Filtration is a widely used method of treating water and recent, numerous attempts aim to develop effective antibacterial composite-based filtration materials [5,6]. Promising examples with respect to their potential application include synthetic polymers (e.g., polypropylene, polyurethane, polyacrylonitrile) [7-9], natural materials (e.g., chitin, chitosan, cellulose, collagen) [10-12] as well as carbon-based composites $[13,14]$, which have been covered with silver nanoparticles (AgNPs) using diverse techniques. The use of silver compounds to disinfect water and the procedure's resulting death of fungi, molds, bacteria and various spores has been documented since ancient times, as cited by Atiyeh [15]. Today, it is a proven fact that direct contact with silver inactivates cells and microorganisms [16]. The mechanism of action of silver involves the inhibition of microbial respiration through binding of metal particles to the bacterial cell membranes [17]. Consequently, silver impairs the microbial respiratory system. The fundamental factors affecting the superior antimicrobial properties of silver-based composites are the size of Ag particles and their solid phase surface development. As recently reported, the "antimicrobial activity of the smaller Ag nanoparticles may be several orders of magnitude greater than that of the corresponding bulk solid" [18]. Thus, it is not surprising that AgNPs are most commonly used in many antibacterial products to protect health and improve the quality of life [19].

Metallization of chitin [20,21], as one of the most abundant structural polysaccharides in nature [22,23], with production in oceans measuring approx. 10 12-14 tons per year [24], remains a solid trend. Chitin is synthesized by a broad assortment of organisms representing different taxonomic groups, mostly crustaceans [25-29] and insects [30-35]. Nowadays, the functionalization of naturally predesigned chitinous scaffolds with a 3D architecture attracts particular attention [36-41]. In this article, the unique skeletal chitin-based 3D scaffolds (Figure 1) isolated from the cultivated under marine farming Aplysina aerophoba marine demosponge were used for the first time as a basic construct for fabrication of an antibacterial water filter. This biomaterial was modified by silver nanoparticle deposition using chemical reduction of silver nitrate and the antibacterial action was investigated. 


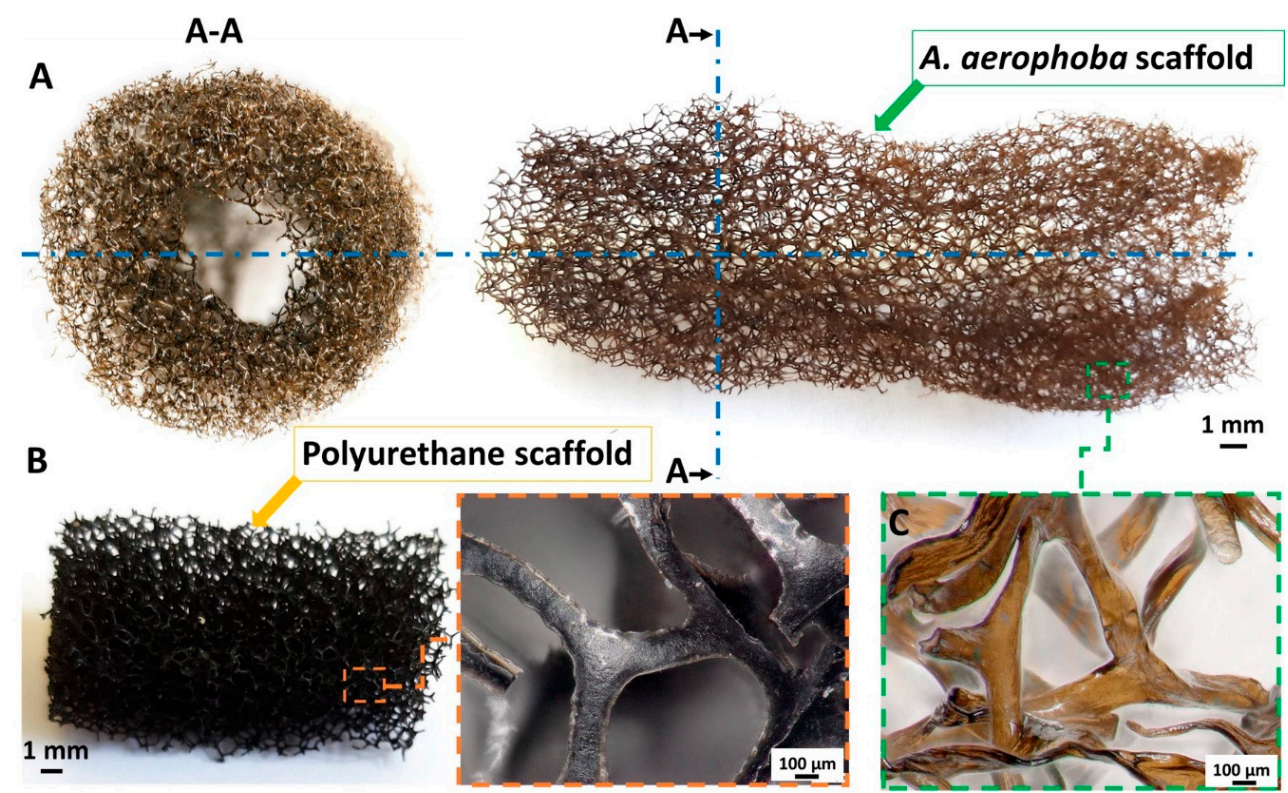

Figure 1. The optical representation of a decellularized Aplysina aerophoba demosponge 3D chitinous skeletal scaffold (A). Representation of the cross-section (A-A). Polyurethane (PU) scaffolds, traditionally used as water filter material, with high magnification of the fibers (B). Microscopic representation of the isolated chitinous skeletal scaffold (C) shows high structural similarity to the commonly used PU-based filtration material (B). The light brownish color is due to the presence of brominated compounds naturally occurring in the skeletal fibers of the sponge.

\section{Results}

Chitinous 3D scaffolds represent an intriguing alternative to synthetic analogues $[20,21,36]$. Due to the high porosity (Figure 2) and structural similarity of the poriferan 3D chitinous scaffolds to synthetically produced porous foams, this biological material is particularly interesting for filtration applications. Based on micro-focused X-ray tomographic (micro-CT) analysis (Figure 2), the porosity of a chitinous scaffold isolated from A. aerophoba demosponge was estimated at $98.5 \%$ (see Table $\mathrm{S} 1$ ).
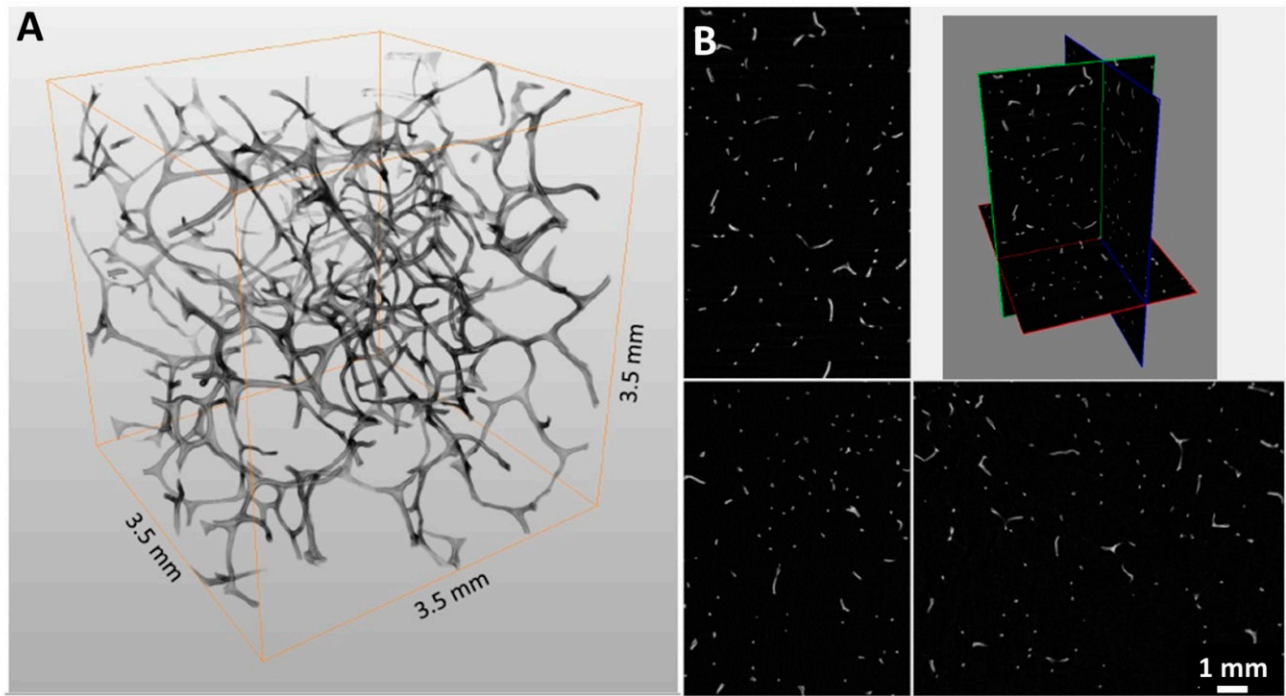

Figure 2. The 3D model (A) and cross-sections (B) of the 3D chitin-Ag/AgBr composite scaffold obtained by micro-CT. 
The alkaline environment of the chemical reduction of $\mathrm{AgNO}_{3}$ promoted the additional release of bromine-derived compounds originally located within the fiber of the chitinous scaffold isolated from A. aerophoba demosponge [37]. The clearly visible metallic layer obtained after metallization with Ag is strongly bound to the chitinous fibers even after ultrasonic treatment (Figure 3).

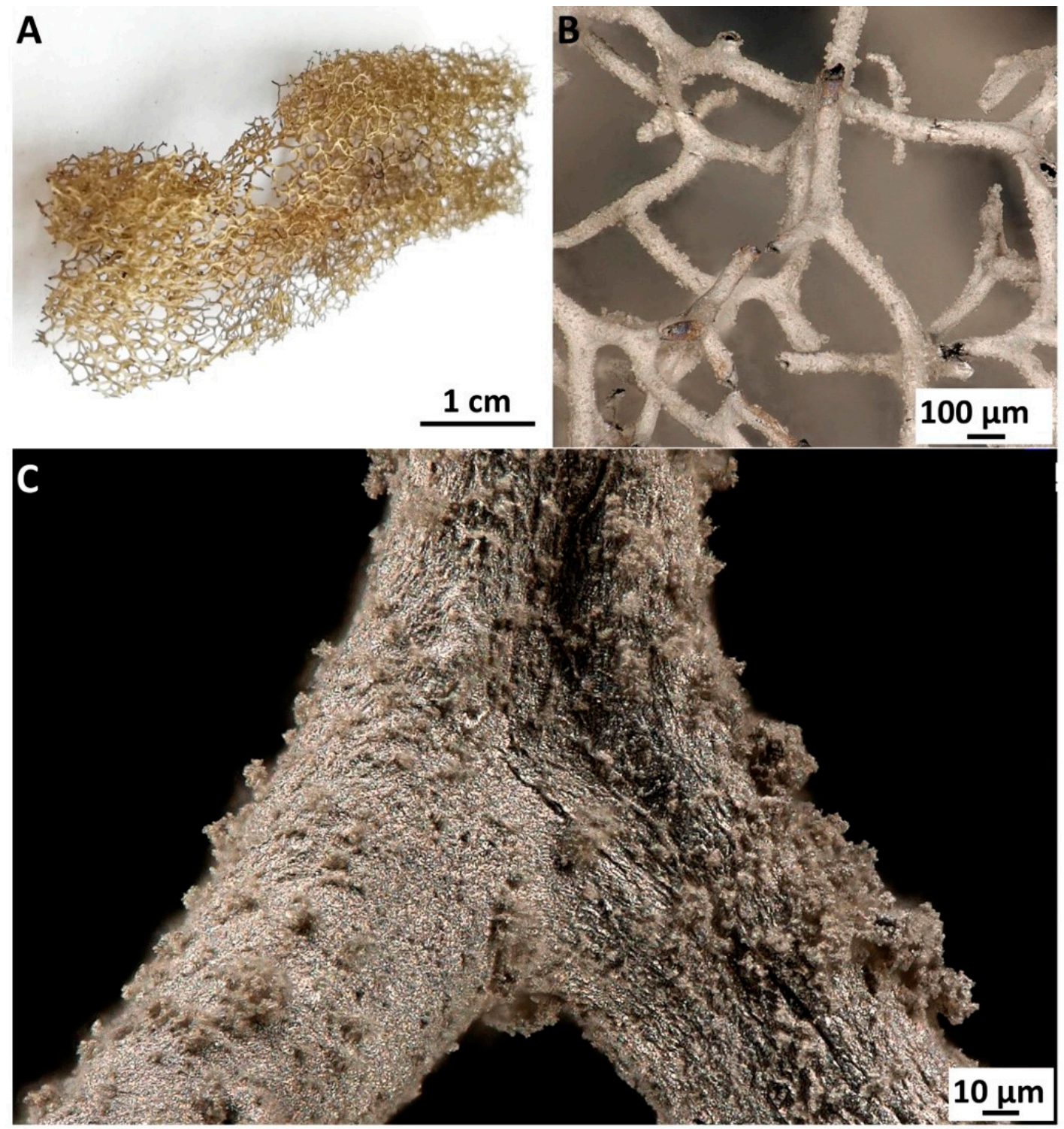

Figure 3. The 3D chitinous scaffolds isolated from the A. aerophoba sponge resemble their microarchitecture being covered with nanoparticles of $\mathrm{Ag} / \mathrm{AgBr}(\mathbf{A}, \mathbf{B})$. The stereomicroscopy image represents the existence of the tightly bound metallized layer, also taken after $30 \mathrm{~min}$ of sonication (C).

$\mathrm{Ag} / \mathrm{AgBr}$ nanoparticles tend to create the spherical-shaped aggregates represented in Figure $4 \mathrm{~A}$. The SEM image in Figure 4A shows the chitinous fiber completely covered by Ag/AgBr aggregates, which are exclusively deposited on the surface of the fibers. The highest fraction of aggregates composed of nanoparticles, which constitute approx. $42 \%$, contains particles with a diameter range of 300-400 nm (see Figure S2). At the surface of chitinous fiber, the creation of nanostructured agglomerates with dimensions up to two $\mu \mathrm{m}$ is also observable. EDX-based analysis of the surface of the metallized A. aerophoba chitinous scaffold confirms domination of Ag and Br (see Figure 4B and Table S2). Obtained data explains the identification of the Ag-bromide phase within the metallized layers using XRD analysis (see Figure 5). 

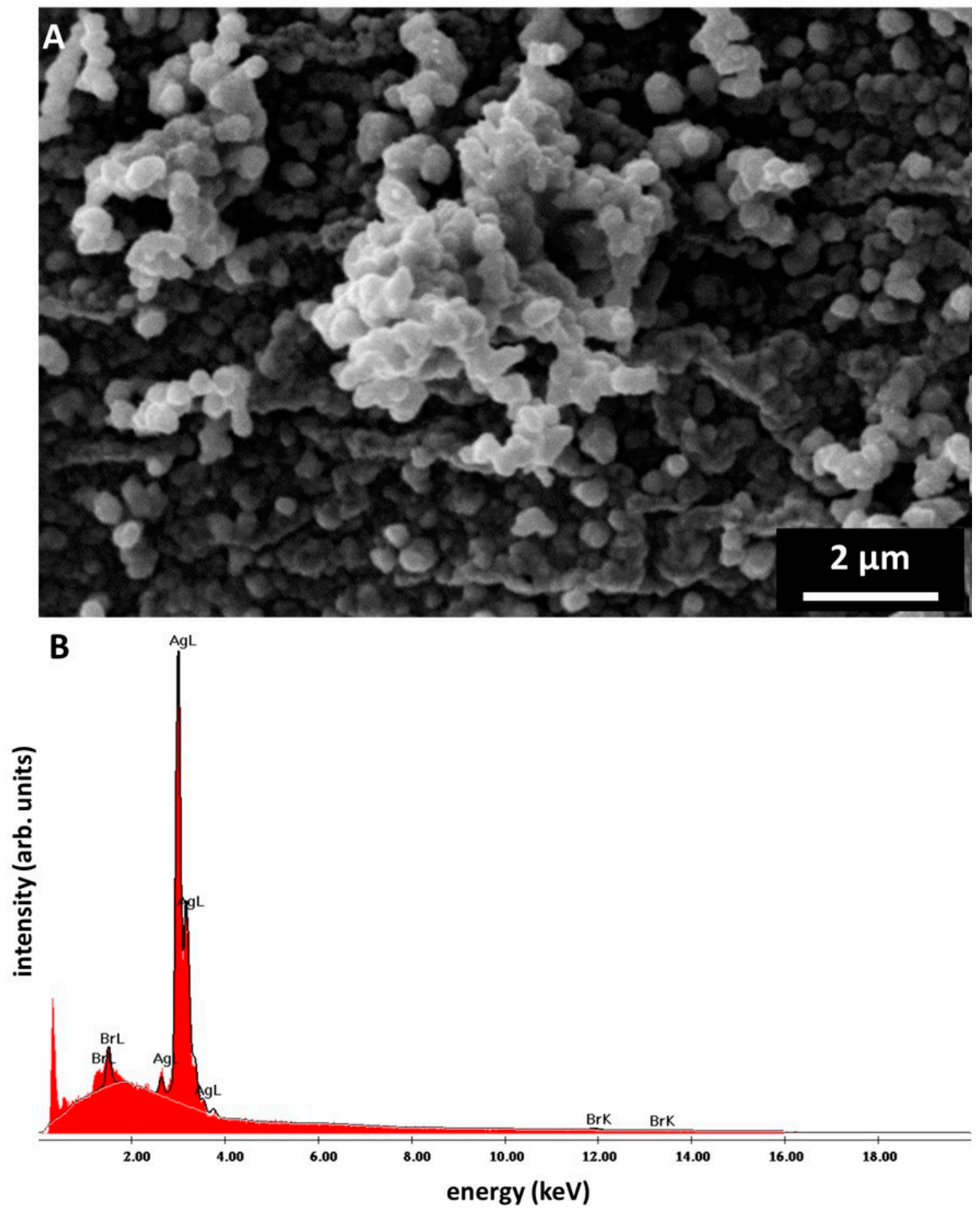

Figure 4. (A) SEM image of the surface of the skeletal chitinous scaffold isolated from A. aerophoba demosponge covered by the layer of silver/silver bromide nanoparticles. EDX analysis confirms the presence of both $\mathrm{Ag}$ and $\mathrm{Br}$ within these nanoparticles (B). This is in good agreement with the XRD data obtained for the same sample (see Figure 5).

The X-ray diffraction patterns of the skeletal chitin sample before and after reaction with $\mathrm{AgNO}_{3}$ solution are shown in Figure 5. In both cases, the crystal structure of chitin (see [42]) is lost due to pretreatment of the samples before XRD. However, some remainders of the chitin structure are still visible, i.e., the diffraction maximum 021 at $2 \theta \approx 12^{\circ}$ and the 'hump' beginning with the 110 reflection near $2 \theta \approx 20^{\circ}$. The XRD line from chitin are marked in Figure 5 . The chitin sample treated with $\mathrm{AgNO}_{3}$ also shows, in addition to the remaining features of the chitin diffraction pattern, peaks of $\mathrm{Ag}$ (PDF\# 04-004-6434) and AgBr (PDF\# 00-006-0438). The presence of metallic Ag confirms the applicability of the synthesis route for the creation of Ag nanoparticles on chitin surface. The Rietveld analysis revealed the Ag lattice parameter of $a=(4.088 \pm 0.001) \AA$, which is practically identical to the tabulated value of $a=4.089 \AA$, and a crystallite size of $D_{\text {iso }}=(13 \pm 2) \mathrm{nm}$, confirming the nanocrystalline 
character of the particles. After Br was identified by the EDX analysis, the remaining peaks in the XRD pattern were successfully assigned to $\mathrm{AgBr}$ crystallizing in space group $\mathrm{Fm} \overline{3} \mathrm{~m}$, with a lattice parameter of $\mathrm{a}=(5.555 \pm 0.002) \AA$. Due to the treatment of the scaffolds with $\mathrm{AgNO}_{3}$, we have also checked for the presence of silver oxides, nitrides and chloride but found no positive match among the database entries. The amount of metallic $\mathrm{Ag}$ is approx. $75 \mathrm{vol} . \%$; the amount of $\mathrm{AgBr}$ is approx. $25 \mathrm{vol} . \%$, as indicated by the quantitative XRD phase analysis.

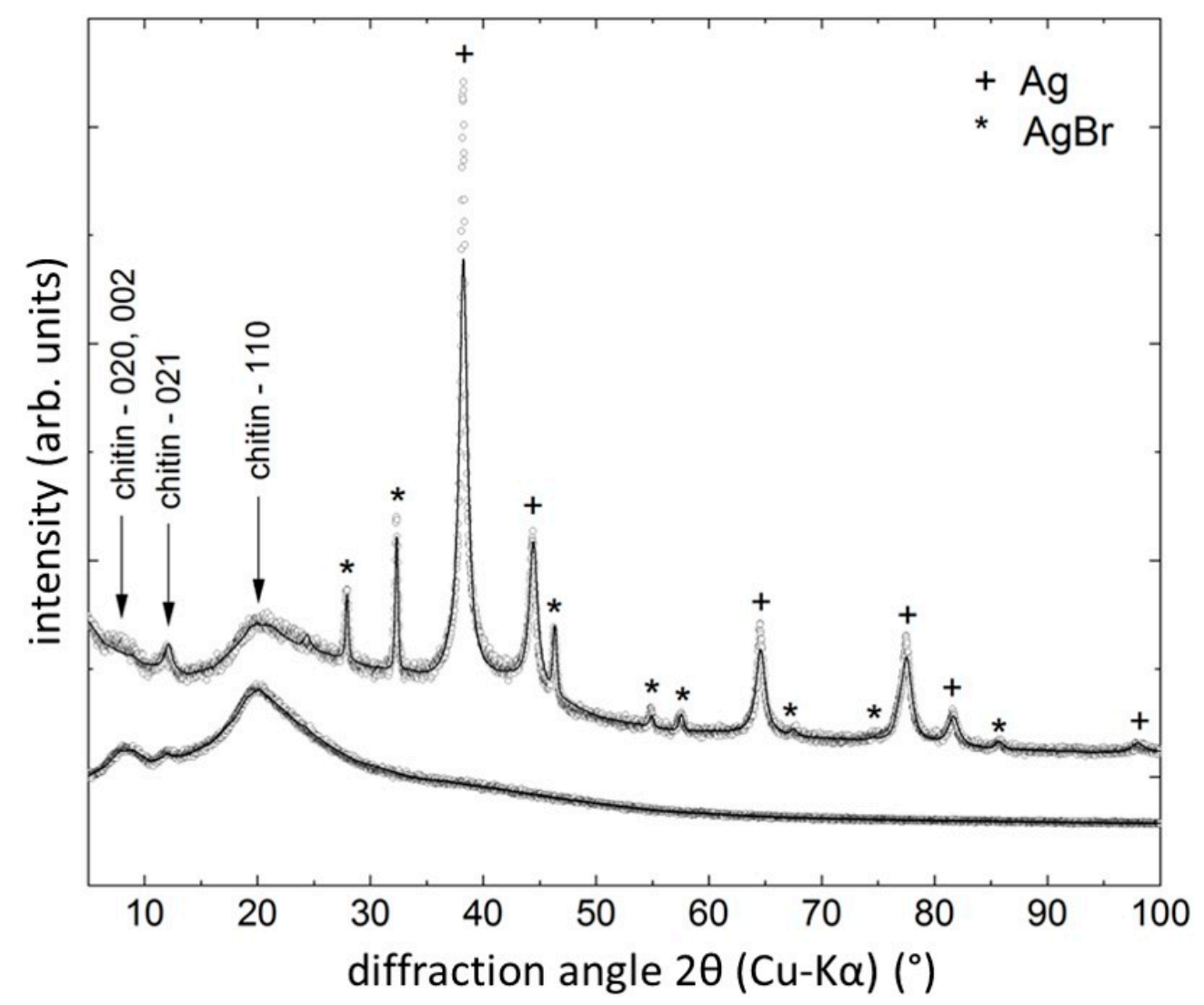

Figure 5. X-ray diffraction patterns (small circles: measured intensities; lines: refinement) of the pure sponge chitin (lower signal intensity) and a chitin sample tightly covered with the Ag and $\mathrm{AgBr}$ nanoparticles (upper signal intensity). The sharp diffraction lines in the diffraction pattern belong either to $\mathrm{Ag}$ or to $\mathrm{AgBr}$, as indicated.

The assessment of the antibacterial activity of the prepared composite was based on the agar diffusion method. As shown in Figure S3, the inhibition zone of the 3D chitin-Ag/AgBr composite in respect to E. coli is greater than that of chitinous skeletal scaffolds before metallization (see Table 1), which indicates the superior antibacterial properties of the created 3D construct. The obtained material contributed to wide zones of inhibition, and the mean value was estimated at 23 and $24 \mathrm{~mm}$ for Escherichia coli and Bacillus subtilis, respectively (see Figure S3). Interesting results were also obtained for the chitinous scaffold before silver coating with respect to the E. coli. The chitinous scaffold used as control was isolated by the method described in Section 4.1. It is mainly composed of Br-containing chitin [37], which is originally responsible for the resistance of this verongiid sponge against predatory microorganisms from marine environments. In this case, the zone of inhibition was $18 \mathrm{~mm}$. However, this effect was not observed in the case of Gram-positive bacterium B. subtilis that was comparatively used in this study. Interestingly, the commercially available antibacterial material Suprasorb ${ }^{\circledR} \mathrm{A}+\mathrm{Ag}$, which was also used for comparative purposes, did not contribute to any zones of inhibition against both bacterial strains studied. 
Table 1. Mean zone of inhibition for both strain ( $\mathrm{mm}$ ) and number of survived Escherichia coli strains after $24 \mathrm{~h}$ of test tube assay.

\begin{tabular}{ccccc}
\hline \multirow{2}{*}{ Material } & \multicolumn{2}{c}{ Agar Diffusion Method } & \multicolumn{2}{c}{ Test Tube Test } \\
\cline { 2 - 5 } & E. coli $(\mathbf{m m})$ & B. subtilis $\mathbf{( m m )}$ & E. coli $(\mathbf{C F U} / \mathbf{1 0 0} \boldsymbol{\mu L})$ & \% of Reduction \\
\hline Chitin-Ag/AgBr scaffold & 23 & 24 & 7 & 99.9 \\
Chitin-based scaffold & 18 & 0 & $\sim 10^{6}$ & 0 \\
Suprasorb ${ }^{\circledR}$ A + Ag & 0 & 0 & $\sim 10^{6}$ & 0 \\
Control & 0 & 0 & $\sim 10^{6}$ & 0 \\
\hline
\end{tabular}

The test tube assay additionally confirmed the antibacterial properties of the chitin-Ag/AgBr scaffold (Figure 6). It was observed that the percentage of the bacteria survival decreased with $24 \mathrm{~h}$ and more than $99.9 \%$ of the initial bacterial CFU was eliminated (for details, see Table 1 and Figure 6 ). The rapid increase in E. coli degradation was observed between 1 and $3 \mathrm{~h}$ (Figure 6A). In contrast to the agar diffusion method, the Br-containing chitinous scaffold before silver coating did not show visible antibacterial potential against $E$. coli even after $24 \mathrm{~h}$.
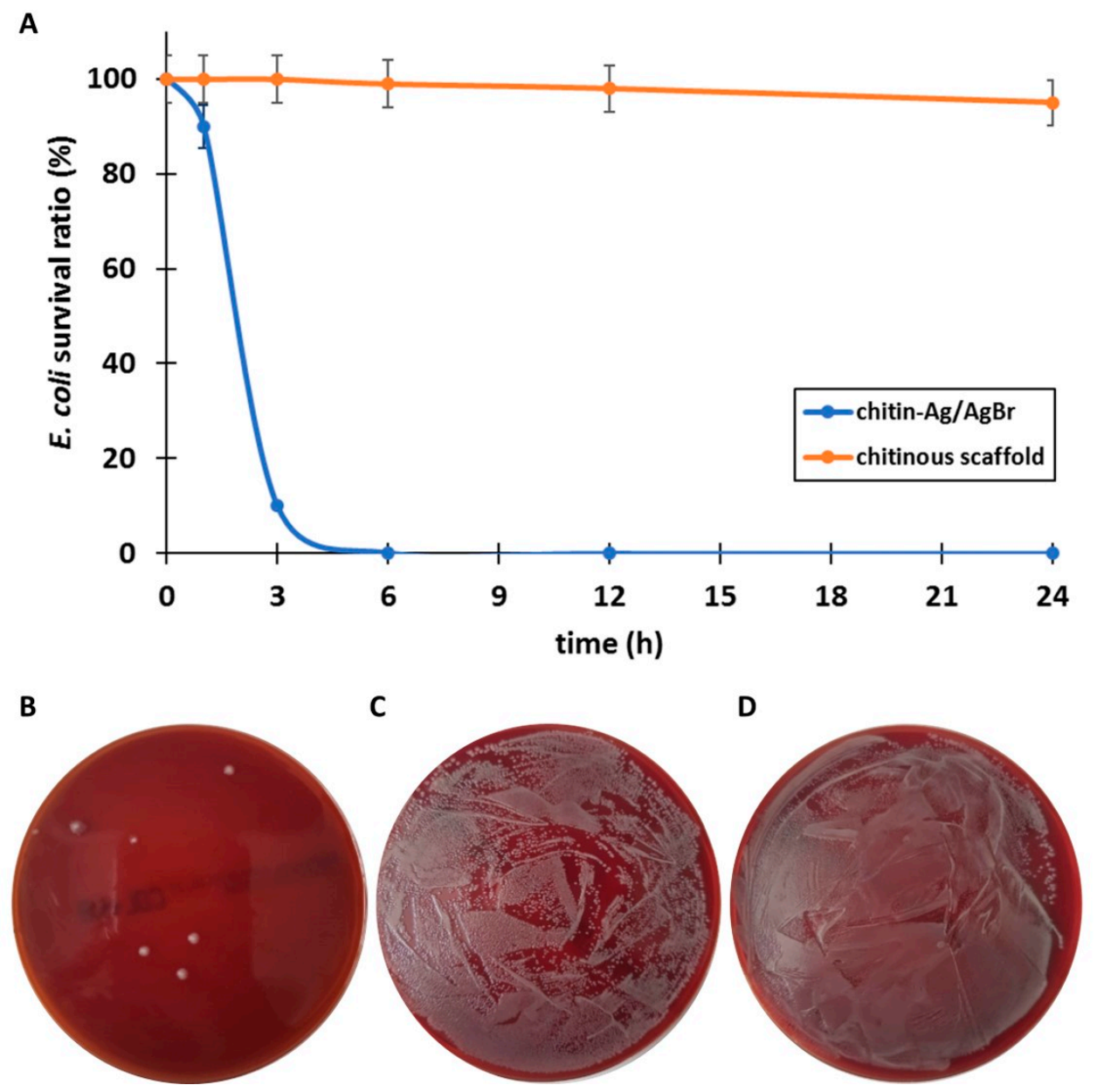

Figure 6. The dynamics of the degradation of live bacteria colonies shown over time, by test tube assay, using a 3D chitin-Ag/AgBr scaffold and a chitinous scaffold before Ag coating, with 5\% error bars (A). Only seven E. coli bacteria colonies survived after $24 \mathrm{~h}$ of testing using a chitin-Ag/AgBr scaffold (B). Both Br-containing chitinous scaffolds before silver coating $(\mathbf{C})$ and commercially available material Suprasorb ${ }^{\circledR} \mathrm{A}+\mathrm{Ag}(\mathrm{D})$ did not show antibacterial activity against $E$. coli even after $24 \mathrm{~h}$. 
Results obtained after filtration (Figure S4) clearly indicate that 3D chitin-Ag/AgBr scaffolds possess antibacterial properties against the E. coli (ATCC ${ }^{\circledR}$ 25922) strain. In experiments, a superior inhibitory effect was observed after $6 \mathrm{~h}$ of filtration with this material. After $24 \mathrm{~h}$, only one colony of this bacterium survived (Figure S4B). Filtration with the Br-containing chitinous scaffold before Ag coating did not cause an observable changes in the number of bacteria. In future, the influence of a diverse chitin- $\mathrm{Ag} / \mathrm{AgBr}$ filter density has to be examined in relation to enhance antibacterial effect. No increase in absorbance at $419 \mathrm{~nm}$ indicated that the silver nanoparticle-based layer was stable on the developed 3D water filter and was not washed away by water flow even after $24 \mathrm{~h} \mathrm{[43].}$

\section{Discussion}

Recently observed rapid development of nanotechnology, especially in the fields of bioinspired materials science and biomimetics, is strictly related to searching for new methods for synthesis of effective hybrid materials and biocomposites with designed properties [44]. Until the discovery of chitin in fibrous skeletons of some verongiid demosponges in 2007 [45], as well as in the verongiid demosponge species A. aerophoba in 2010 [46], the commonly accepted opinion was that skeletal fibers of these demosponges are made of a proteinaceous, biological material called spongin [47]. However, this erroneous assumption was experimentally proved based on the solubility of spongin in alkaline solutions, which was not observed with chitin resistant to such chemical conditions $[20,36]$. It is the resistance of chitin to an alkaline medium up to a concentration of $10 \%$ that allows us to look for key ways to use it in specific chemical reactions, including silver metallization, as described in this work. For example, the implementation of such a reaction using spongin-based matrices would not have been possible.

Chitin of poriferan origin also possess characteristic features such as thermostability up to $400{ }^{\circ} \mathrm{C}[41,48,49]$, cytocompatibility [50,51] and microporosity [36,52]. Recently, practical applications of demosponge chitinous scaffolds were reported for tissue engineering [39,50,51,53], uranium adsorption [54], bioelectrometallurgy and extreme biomimetics [21] as well as for the photodegradation of organic dyes [49]. Here, we describe, for the first time, antibacterial properties of composite material in the form of 3D chitin-based skeletal scaffolds covered with AgNPs and Ag-bromide. The formation of Ag-bromide on the surface of this specific chitin is due to the presence of bromine in the chitin skeleton of these sponges. We suggest that this compound is formed as a result of alkaline extraction of bromine-containing compounds in the presence of silver ions.

Originally, diverse brominated derivatives (mostly bromotyrosines) [37] are located in the skeletal fibers being intercalated into chitinous layers. They represent an effective form of biochemical defense against harmful pathogens, which constantly fall inside the verongiid sponge, which filters the surrounding water to extract the appropriate feed (i.e., viruses, bacteria, organic micro debris). These unique defense strategies, based on Br-containing secondary metabolite production, allowed this organism to survive more than 500 millions of years [37]. Today, bromotyrosines are recognized as multi-targeted marine drugs with broad fields of applications (i.e., as antitumorigenic and antimetastatic agents $[55,56])$. In order to isolate pure chitinous scaffolds from verongiid demosponges, diverse methodological approaches based on chemical, electrochemical and enzymatic treatments were reported (see for overview [36,57]). Nonetheless, the alkali treatment of verongiid skeletons is most commonly used $[20,36,40]$ and can be regulated with respect to residual bromine concentration in the chitinous matrices. In our case, secondary metabolite compounds present within scaffolds become a great source of natural bromine. Previously, superb antibacterial properties of Ag [6,16,58] and AgBr [59-61] nanoparticles were already reported, but as separate substances and not within composite materials.

In order to determine antibacterial properties of the designed 3D chitin-Ag/AgBr composite, the agar diffusion method as well as test tube assay was carried out. Moreover, a simple prototype of the filtration set was proposed to assess the determination ability of 3D chitin- $\mathrm{Ag} / \mathrm{AgBr}$ scaffolds in terms of E. coli inactivation. Based on the obtained data, appropriative antibacterial properties against the E. coli strain were reported here. Only one bacteria colony from $10^{6} \mathrm{CFU} / \mu \mathrm{L}$ survived after $24 \mathrm{~h}$ of filtration. 
In the 3D composite scaffold developed in this study, under ambient conditions, the content of $\mathrm{Ag}$ is approx. $75 \mathrm{vol} . \%$ and the amount of $\mathrm{AgBr}$ is approx. $25 \mathrm{vol} . \%$. This distinguishes the method described in this study from the metallization of chitin in harsh chemical conditions. For example, previously, poriferan chitin was effectively used as a template for solvothermal and hydrothermal conditions according to an extreme biomimetic approach $[41,44,48,49,62]$. Element oxide-based composites such as chitin- $\mathrm{SiO}_{2}[62,63]$, chitin- $\mathrm{GeO}_{2}$ [41], chitin- $\mathrm{ZrO}_{2}$ [48,64], chitin- $\mathrm{ZnO}$ [65], and chitin-hematite [66] were synthesized under hydrothermal conditions between 65 and $185^{\circ} \mathrm{C}$.

\section{Materials and Methods}

\subsection{Chitin Scaffold Isolation}

Air-dried specimens of cultivated Aplysina aerophoba (Nardo, 1833) marine demosponges were purchased from BromMarin $\mathrm{GmbH}$ (Freiberg, Germany). Chitinous scaffolds were isolated by chemical treatment of specimens in 2 days as follows. At the first stage, selected fragments of A. aerophoba skeleton were immersed in pure distilled water at $80^{\circ} \mathrm{C}$ for $24 \mathrm{~h}$ to remove water-soluble compounds and cells, which were disrupted due to osmotic shock. In the next step, cell-free skeletons, were immersed in $20 \%$ acetic acid at room temperature over $4 \mathrm{~h}$ in order to remove calcium carbonates. The prefinal stage included treatment with $2.5 \mathrm{M} \mathrm{NaOH}$ solution for $6 \mathrm{~h}$ at $37^{\circ} \mathrm{C}$, for deproteinization and partial depigmentation. Finally, Br-containing skeletal 3D chitinous scaffolds were neutralized by distilled water and stored at $4{ }^{\circ} \mathrm{C}$.

\subsection{Fabrication of Silver-Coated 3D Chitinous Scaffolds}

In order to obtain AgNps on the surface of skeletal chitinous scaffolds isolated from the cultivated A. aerophoba demosponge, chemical reduction of $\mathrm{AgNO}_{3}$ was carried out. For this purpose, a selected fragment of the skeletal scaffold $(10 \times 30 \mathrm{~mm})$ was immersed in $30 \mathrm{~mL}$ of $1 \mathrm{M} \mathrm{AgNO}_{3}$ solution for $1 \mathrm{~h}$ at room temperature. Then, $15 \mathrm{~mL}$ of $0.8 \mathrm{M} \mathrm{NaOH}$ was added to the above mixture and formation of a precipitate was observed. In the next step, a concentrated ammonia solution was added dropwise until total precipitate dissolution was achieved. After $15 \mathrm{~min}, 1 \mathrm{~mL}$ of ethanol and $25 \mathrm{~mL}$ of a mixture of $0.08 \mathrm{M}$ glucose and $0.04 \mathrm{M}$ citric acid were added. The obtained construct was washed several times by deionized water and treated by ultrasound $(60 \mathrm{kHz}, 300 \mathrm{~W})$ for $30 \mathrm{~min}$ at room temperature in order to remove non-attached nanoparticles of silver.

\subsection{Characterization of Obtained Materials}

\subsubsection{Digital Microscopy}

Corresponding samples were observed and analyzed by an advanced imaging and measurement system consisting of a Keyence VHX-6000 digital optical microscope (Osaka, Japan) and the swing-head zoom lenses VH-Z20R (magnification up to 200X) and VH-Z100UR (magnification up to 1000X).

\subsubsection{Micro-CT Analysis}

Scaffolds were scanned using a micro-focused X-ray tomographic system (MICRO XCT-400, Xradia-Zeiss, Pleasanton, CA, USA) at $40 \mathrm{kV}$ and $200 \mu$ A. For each sample, 1500 projection images were recorded with an exposure time of $12 \mathrm{sec}$ and a magnification objective of $20 \mathrm{x}$. The volume was reconstructed with the instrument software and was then exported to the CTAn (Bruker Billerica, MA, USA) program for further 3D image analysis. Voxel size was the same for all samples $(2 \times 2 \times 2 \mu \mathrm{m})$.

\subsubsection{Infrared Spectroscopy}

Attenuated total reflectance Fourier transform infrared spectroscopy (ATR-FTIR), was used for the qualitative characterization and identification of the isolated materials. The samples were analyzed by a Nicolet 210c spectrometer (Thermo Fisher Scientific, Waltham, MA, USA). 


\subsubsection{UV-VIS Spectroscopy}

The absorption at $419 \mathrm{~nm}$ was recorded with a spectrophotometer (SPECORD S10, Carl Zeiss, Germany). Measurements were recorded for determination of the presence of silver nanoparticles in the solution after filtration [43]. A quartz cuvette was used with a path length of $1 \mathrm{~cm}$ (Quartz SUPRASIL ${ }^{\circledR}$, Hellma Analytics, Müllheim, Germany) and operated at a resolution of $5 \mathrm{~nm}$.

\subsubsection{Scanning Electron Microscopy (SEM) and Energy-Dispersive X-ray Spectroscopy (EDX)}

Samples were prepared for analysis by freeze-drying, followed by covering with Au using a Cressington Sputter Coater S150B (BOC Edwards, Wilmington, MA, USA). Scanning electron microscopy was performed using a Hitachi S-4700-II (Hitachi, Ltd., Tokyo, Japan) field emission microscope. The elements were analyzed by energy-dispersive X-ray spectroscopy in the EDX analysis system from EDAX and a XL30ESEM Philips scanning electron microscope (Philips, Amsterdam, The Netherlands). Observations were carried out under high vacuum, $20 \mathrm{kV}$ voltage, and $6500 \times$ and $8000 \times$ magnification. The particle diameter was determined from 100 measurements using representative micrographs by the software (ImageJ, National Institutes of Health, Bethesda, MD, USA).

\subsubsection{X-ray Diffraction}

X-ray diffraction measurements were performed with the purpose of phase identification and quantification. A FPM RD7 diffractometer equipped with a sealed X-ray tube with $\mathrm{Cu}$ anode operated at $40 \mathrm{kV} / 30 \mathrm{~mA}$ was used. The diffractometer worked in the symmetrical diffraction geometry and the powderized sample was fixed with ethanol on a Si[510]-cut zero-background holder. The diffracted beam passed a graphite monochromator to eliminate unwanted radiation components before being registered by a proportional counter. The measurements covered the angular range from $5^{\circ}$ to $150^{\circ} 2 \theta$, with a step size of $0.02^{\circ}$ and a dwell time of $12 \mathrm{~s}$ per point. Data analysis was performed by database search (ICSD PDF-4+) with the Panalytical HighScore+ program. Rietveld refinement of the diffraction patterns was performed by the MAUD software package [67].

\subsection{Antibacterial Activity Studies}

\subsubsection{Determination of the Zone of Inhibition}

The chitin-Ag/AgBr (1) (Figure S2) composite scaffolds were tested for their antibacterial activity using an agar diffusion method. For this purpose, $0.01 \mathrm{~g}$ of sample was prepared. For comparison, chitinous scaffold before silver coating (2) and commercially available antibacterial wound dressing Suprasorb ${ }^{\circledR}$ A + Ag (3) were used. E. coli (ATCC ${ }^{\circledR}$ 25922) and B. subtilis B9 (Collection of Department of Epizootiology and Clinic of Infectious Diseases, University of Life Sciences in Lublin, Poland) were taken as model Gram-negative and Gram-positive bacteria (1.5 on the McFarland scale). Bacteria strain was applied with agar Mueller-Hinton medium. Afterwards, plates were incubated at $37^{\circ} \mathrm{C}$ for $24 \mathrm{~h}$. The diameter of inhibitory zone surrounding material pieces was measured in $\mathrm{mm}$ for each specimen. Tests performed on three agar plates (for each material) determined a mean value.

\subsubsection{Test Tube Antibacterial Assay}

For the test tube assay, the E. coli ATCC ${ }^{\circledR} 25,922$ suspension ( 0.8 on the McFarland scale, approx. $10^{6} \mathrm{CFU} / \mu \mathrm{L}$ ) in $0.9 \%$ sodium chloride was prepared in sterilized test tubes. An amount of $0.01 \mathrm{~g}$ of sample (No. 1-3 see Section 4.4.1.) was put into the separated test tubes and immersed in $1.5 \mathrm{~mL}$ of suspension described above at $37^{\circ} \mathrm{C}$ for $1,3,6,12$ and $24 \mathrm{~h}$. The scaffolds were taken out from the test tubes. Then, $100 \mu \mathrm{L}$ of suspension was plated on Columbia LabAgar plates $+5 \%$ sheep blood and incubated at $37^{\circ} \mathrm{C}$ for $24 \mathrm{~h}$ for determination of number of survive bacterial colonies from the control sample. These measurements were repeated three times and a mean value was evaluated. 


\subsubsection{Determination of Antibacterial Properties-Filtration Test}

The antibacterial activity of the obtained chitin- $\mathrm{Ag} / \mathrm{AgBr}(1)$ was also determined using the filtration method. The E. coli ATCC ${ }^{\circledR} 25,922$ suspension $\left(0.8\right.$ on the McFarland scale, approx. $\left.10^{6} \mathrm{CFU} / \mu \mathrm{L}\right)$ was kept in a storage $1000 \mathrm{~mL}$ container filled with $0.9 \%$ sodium chloride and filtered at a flow rate of $330 \mathrm{~mL} / \mathrm{min}$. The suspension was pumped into the sterilized filtration cartridge, which consists of a $0.5 \mathrm{~g}$ of filter inside $50 \mathrm{~cm}^{3}$ falcon tubes (Figure S1). Changes in the survival rate of the bacteria colony were determined for 1, 3, 6, 12 and $24 \mathrm{~h}$ analogous to the test described above.

\section{Conclusions}

The fundamental difference between chitin-based 3D scaffolds originating from verongiid sponges and other, previously reported chitinous matrices is the possibility to regulate the content of $\mathrm{Br}$ during the preparation process by changing the time of alkaline treatment of original skeletal 3D constructs. Therefore, the experimental approach described in this study enables unique nanostructured $\mathrm{Ag} / \mathrm{AgBr}$ composite in the form of a nanolayer, which remains strongly bound to the surface of the organic matrix and is responsible for selective antibacterial activities. The results open the path to using chitin-based skeletal matrices in the form of acellular scaffolds. Due to the natural ability of the A. aerophoba demosponges to regenerate their skeletons and to grow at low depths under marine farming conditions at large scales, their potential for applications in bioinspired materials science and technologies increases dramatically. Future research, dedicated to the optimization of such naturally derived, already prefabricated materials using other metal ions as well as alternative reductants (e.g., lignosulfonates) also for special biomedical applications is strongly indicated.

Supplementary Materials: The following are available online at http://www.mdpi.com/1660-3397/18/6/304/s1, Figure S1: The protype of the filtration set used in this study. Figure S2: Percentage of Ag/AgBr aggregates in individual fractions as a function of diameter ranges. Figure S3: Agar diffusion method results. The antimicrobial activity of chitin-Ag/AgBr scaffolds (1), chitinous scaffold before metallization (2) against E. coli (A). Lack of antimicrobial activity represent by Suprasorb ${ }^{\circledR} \mathrm{A}+\mathrm{Ag}$ (3) against E. coli (A). The antimicrobial activity of chitin-Ag/AgBr scaffold (1) against B. subtilis (B). Figure S4: Dynamics of the degradation of live bacteria colonies as a function of time of filtration with $5 \%$ error bars (A). Filtration clearly indicates that chitin-Ag/AgBr scaffolds possess antibacterial properties against the E. coli (ATCC $\left.{ }^{\circledR} 25922\right)$ strain (blue line). Br-containing chitinous scaffolds before AgNP coating did not reflect any antibacterial effect. The number of survived bacteria colonies was uncountable, even after $24 \mathrm{~h}$ (orange line). Only one colony of E. coli survived after $24 \mathrm{~h}$ of filtration using chitin-Ag/AgBr scaffold (B). Chitinous scaffold before silver coating did not show antibacterial potential against E. coli even after $24 \mathrm{~h}(\mathrm{C})$. Table S1: Results of 3D quantitative analysis (micro-CT). Table S2. Results of local chemical analyses (SEM-EDX) of selected areas of the A. aerophoba chitinous scaffold after metallization.

Author Contributions: Conceptualization, V.K., M.R.-N. and H.E.; data curation, M.R.-N.; investigation, T.M., M.C., H.M., C.S., J.Z., B.D., A.V., J.J. and A.F.; methodology, T.M., M.C., H.M., C.S., D.R., Ł.A., V.K. and D.S.; resources, J.Z., B.D., A.V. and D.S.; software, I.P. and J.J.; supervision, T.J.; validation, I.P., D.R. and T.J.; visualization, Ł.A.; writing-original draft, T.M., N.B. and H.E. All authors have read and agreed to the published version of the manuscript.

Funding: This work was partially supported by the DFG Project HE 394/3, SMWK Project no. 02010311 (Germany), as well as by the Ministry of Science and Higher Education (Poland). M.R.N. is supported by Alexander von Humboldt Fellowship. T.M. is supported by DAAD (Personal ref. no. 91734605).

Acknowledgments: Special thank for Anna Wilczyńska for preparing the agar plate photos.

Conflicts of Interest: The authors declare no conflict of interest.

\section{References}

1. Batra, S.; Adhikari, P.; Ghai, A.; Sharma, A.; Sarma, R.; Suneetha, V. Study and design of portable antimicrobial water filter. Asian J. Pharm. Clin. Res. 2017, 10, 268. [CrossRef]

2. Khan, S.T.; Malik, A. Engineered nanomaterials for water decontamination and purification: From lab to products. J. Hazard. Mater. 2019, 5, 295-308. [CrossRef] [PubMed]

3. Fewtrell, L. Silver: Water Disinfection and Toxicity; Centre for Research into Environment and Health, World Health Organization: Geneva, Switzerland, 2014; pp. 1-50. 
4. Wang, L.; Hu, C.; Shao, L. The antimicrobial activity of nanoparticles: Present situation and prospects for the future. Int. J. Nanomed. 2017, 12, 1227-1249. [CrossRef] [PubMed]

5. Pronk, W.; Ding, A.; Morgenroth, E.; Derlon, N.; Desmond, P.; Burkhardt, M.; Wu, B.; Fane, A.G. Gravity-driven membrane filtration for water and wastewater treatment: A review. Water Res. 2019, 149, 553-565. [CrossRef] [PubMed]

6. Karumuri, A.K.; Oswal, D.P.; Hostetler, H.A.; Mukhopadhyay, S.M. Silver nanoparticles attached to porous carbon substrates: Robust materials for chemical-free water disinfection. Mater. Lett. 2013, 109, 83-87. [CrossRef]

7. Phong, N.T.P.; Thanh, N.V.K.; Phuong, P.H. Fabrication of antibacterial water filter by coating silver nanoparticles on flexible polyurethane foams. J. Phys. Conf. Ser. 2009, 187, 012079. [CrossRef]

8. Picca, R.A.; Paladini, F.; Sportelli, M.C.; Pollini, M.; Giannossa, L.C.; Di Franco, C.; Panico, A.; Mangone, A.; Valentini, A.; Cioffi, N. Combined approach for the development of efficient and safe nanoantimicrobials: The case of nanosilver-modified polyurethane foams. ASC Biomater. Sci. 2017, 3, 1417-1425. [CrossRef]

9. Shi, Y.; Li, Y.; Zhang, J.; Yu, Z.; Yang, D. Electrospun polyacrylonitrile nanofibers loaded with silver nanoparticles by silver mirror reaction. Mater. Sci. Eng. C 2015, 51, 346-355. [CrossRef] [PubMed]

10. Heinemann, S.; Heinemann, C.; Ehrlich, H.; Meyer, M.; Baltzer, H.; Worch, H.; Hanke, T. A novel biomimetic hybrid material made of silicified collagen: Perspectives for bone replacement. Adv. Eng. Mater. 2007, 9, 1061-1068. [CrossRef]

11. Cooper, A.; Oldinski, R.; Ma, H.; Bryers, J.D.; Zhang, M. Chitosan-based nanofibrous membranes for antibacterial filter applications. Carbohydr. Polym. 2013, 30, 254-259. [CrossRef] [PubMed]

12. Jain, S.; Bhanjana, G.; Heydarifard, S.; Dilbaghi, N.; Nazhad, M.M.; Kumar, V.; Kim, K.; Kumar, S. Enhanced antibacterial profile of nanoparticle impregnated cellulose foam filter paper for drinking water filtration. Carbohydr. Polym. 2018, 202, 219-226. [CrossRef] [PubMed]

13. Karwowska, E. Antibacterial potential of nanocomposite-based materials-A short review. Nanotechnol. Rev. 2017, 6, 243-254. [CrossRef]

14. Zeng, X.; Mccarthy, D.T.; Deletic, A.; Zhang, X. Silver/reduced graphene oxide hydrogel as novel bactericidal filter for point-of-use water disinfection. Adv. Funct. Mater. 2015, 25, 4344-4351. [CrossRef]

15. Atiyeh, B.S.; Costagliola, M.; Hayek, S.N.; Dibo, S.A. Effect of silver on burn wound infection control and healing: Review of the literature. Burns 2007, 33, 139-148. [CrossRef] [PubMed]

16. Cho, K.-H.; Park, J.-E.; Osaka, T.; Park, S.-G. The study of antimicrobial activity and preservative effects of nanosilver ingredient. Electrochim. Acta 2005, 51, 956-960. [CrossRef]

17. Imani, R.; Talaiepour, M.; Dutta, J.; Ghobadinezhad, M.R.; Hemmasi, A.H.; Nazhad, M.M. Production of antibacterial filter paper from wood cellulose. BioResources 2011, 6, 891-900.

18. Nguyen, V.Q.; Ishihara, M.; Nakamura, S.; Hattori, H.; Ono, T.; Miyahira, Y.; Matsui, T. Interaction of silver nanoparticles and chitin powder with different sizes and surface structures: The correlation with antimicrobial activities. J. Nanomater. 2013, 2013, 467534. [CrossRef]

19. Klippstein, R.; Fernandez-Montesinos, P.M.; Castillo, R.; Zaderenko, A.P.; Pozo, D. Silver nanoparticles interactions with the immune system: Implications for health and disease. In Silver Nanoparticles; Pozo, D., Ed.; InTech: Rijeka, Croatia, 2010; pp. 310-325.

20. Wysokowski, M.; Petrenko, I.; Stelling, A.L.; Stawski, D.; Jesionowski, T.; Ehrlich, H. Poriferan chitin as a versatile template for extreme biomimetics. Polymers 2015, 7, 235-265. [CrossRef]

21. Petrenko, I.; Bazhenov, V.V.; Galli, R.; Wysokowski, M.; Fromont, J.; Schupp, P.J.; Stelling, A.L.; Niederschlag, E.; Stöker, H.; Kutsova, V.Z.; et al. Chitin of poriferan origin and the bioelectrometallurgy of copper/copper oxide. Int. J. Biol. Macromol. 2017, 104, 1626-1632. [CrossRef] [PubMed]

22. Crini, G. Historical review on chitin and chitosan biopolymers. Environ. Chem. Lett. 2019, 17, 1623-1643. [CrossRef]

23. Ehrlich, H. Chitin and collagen as universal and alternative templates in biomineralization. Int. Geol. Rev. 2010, 52, 661-699. [CrossRef]

24. Yadav, M.; Goswami, P.; Paritosh, K.; Kumar, M.; Pareek, N.; Vivekanand, V. Seafood waste: A source for preparation of commercially employable chitin/chitosan materials. Bioresour. Bioprocess. 2019, 6, 8. [CrossRef]

25. Duan, B.; Huang, Y.; Lu, A.; Zhang, L. Recent advances in chitin based materials constructed via physical methods. Prog. Polym. Sci. 2018, 82, 1-33. [CrossRef] 
26. Zhang, J.; Feng, M.; Lu, X.; Shi, C.; Li, X.; Xin, J.; Yue, G.; Zhang, S. Base-free preparation of low molecular weight chitin from crab shell. Carbohydr. Polym. 2018, 190, 148-155. [CrossRef] [PubMed]

27. Hamed, I.; Özogul, F.; Regenstein, J.M. Industrial applications of crustacean by-products (chitin, chitosan, and chitooligosaccharides): A review. Trends Food Sci. Technol. 2016, 48, 40-50. [CrossRef]

28. Seear, P.J.; Tarling, G.A.; Burns, G.; Goodall-Copestake, W.P.; Gaten, E.; Özkaya, Ö.; Rosato, E. Differential gene expression during the moult cycle of Antarctic krill (Euphausia superba). BMC Genom. 2010, 11, 582. [CrossRef] [PubMed]

29. Zeng, J.B.; He, Y.S.; Li, S.L.; Wang, Y.Z. Chitin whiskers: An overview. Biomacromolecules 2012, 13, 1-11. [CrossRef] [PubMed]

30. Chandran, R.; Williams, L.; Hung, A.; Nowlin, K.; Lajeunesse, D. SEM characterization of anatomical variation in chitin organization in insect and arthropod cuticles. Micron 2016, 82, 74-85. [CrossRef] [PubMed]

31. Merzendorfer, H.; Zimoch, L. Chitin metabolism in insects: Structure, function and regulation of chitin synthases and chitinases. J. Exp. Biol. 2003, 206, 4393-4412. [CrossRef] [PubMed]

32. Vincent, J.F.V.; Wegst, U.G.K. Design and mechanical properties of insect cuticle. Arthropod Struct. Dev. 2004, 33, 187-199. [CrossRef] [PubMed]

33. Rudall, K.M. The chitin/protein complexes of insect cuticles. Adv. Insect Phys. 1963, 1, 257-313.

34. Liu, S.; Sun, J.; Yu, L.; Zhang, C.; Bi, J.; Zhu, F.; Qu, M.; Jiang, C.; Yang, Q. Extraction and characterization of chitin from the beetle Holotrichia parallela motschulsky. Molecules 2012, 17, 4604-4611. [CrossRef] [PubMed]

35. Davies, G.J.G.; Knight, D.P.; Vollrath, F. Chitin in the silk gland ducts of the spider Nephila edulis and the silkworm Bombyx mori. PLoS ONE 2013, 8, e73225. [CrossRef] [PubMed]

36. Klinger, C.; Żółtowska-Aksamitowska, S.; Wysokowski, M.; Tsurkan, M.V.; Galli, R.; Petrenko, I.; Machałowski, T.; Ereskovsky, A.; Martinović, R.; Muzychka, L.; et al. Express method for isolation of ready-to-use 3D chitin scaffolds from Aplysina archeri (Aplysineidae: Verongiida) Demosponge. Mar. Drugs 2019, 17, 131. [CrossRef] [PubMed]

37. Kovalchuk, V.; Voronkina, A.; Binnewerg, B.; Schubert, M.; Muzychka, L.; Wysokowski, M.; Tsurkan, M.V.; Bechmann, N.; Petrenko, I.; Fursov, A.; et al. Naturally drug-loaded chitin: Isolation and applications. Mar. Drugs 2019, 17, 574. [CrossRef] [PubMed]

38. Binnewerg, B.; Schubert, M.; Voronkina, A.; Muzychka, L.; Wysokowski, M.; Petrenko, I.; Djurović, M.; Kovalchuk, V.; Tsurkan, M.; Martinovic, R.; et al. Marine biomaterials: Biomimetic and pharmacological potential of cultivated Aplysina aerophoba marine demosponge. Mater. Sci. Eng. C 2020, 109, 110566. [CrossRef] [PubMed]

39. Schubert, M.; Binnewerg, B.; Voronkina, A.; Muzychka, L.; Wysokowski, M.; Petrenko, I.; Kovalchuk, V.; Tsurkan, M.; Martinovic, R.; Bechmann, N.; et al. Naturally prefabricated marine biomaterials: Isolation and applications of flat chitinous 3D scaffolds from Ianthella labyrinthus (Demospongiae: Verongiida). Int. J. Mol. Sci. 2019, 20, 5105. [CrossRef] [PubMed]

40. Wysokowski, M.; Machałowski, T.; Petrenko, I.; Schimpf, C.; Rafaja, D.; Galli, R.; Zietek, J.; Pantovic, S.; Voronkina, A.; Ivanenko, V.K.V.N.; et al. 3D chitin scaffolds of marine Demosponge origin for biomimetic mollusk hemolymph-associated biomineralization ex-vivo. Mar. Drugs 2020, 18, 123. [CrossRef] [PubMed]

41. Wysokowski, M.; Motylenko, M.; Beyer, J.; Makarova, A.; Stöcker, H.; Walter, J.; Galli, R.; Kaiser, S.; Vyalikh, D.; Bazhenov, V.V.; et al. Extreme biomimetic approach for developing novel chitin- $\mathrm{GeO}_{2}$ nanocomposites with photoluminescent properties. Nano Res. 2015, 8, 2288-2301. [CrossRef]

42. Machałowski, T.; Wysokowski, M.; Tsurkan, M.V.; Galli, R.; Żółtowska-Aksamitowska, S.; Petrenko, I.; Czaczyk, K.; Kraft, M.; Bertau, M.; Bechmann, N.; et al. Spider chitin: An ultrafast microwave-assisted method for chitin isolation from Caribena versicolor spider molt cuticle. Molecules 2019, 24, 3736. [CrossRef]

43. Jain, P.; Pradeep, T. Potential of silver nanoparticle-coated polyurethane foam as an antibacterial water filter. Biotechnol. Bioeng. 2005, 90, 59-63. [CrossRef] [PubMed]

44. Wysokowski, M.; Behm, T.; Born, R.; Bazhenov, V.V.; Meißner, H.; Richter, G.; Szwarc-Rzepka, K.; Makarova, A.; Vyalikh, D.; Schupp, P.; et al. Preparation of chitin-silica composites by in vitro silicification of two-dimensional Ianthella basta demosponge chitinous scaffolds under modified Stöber conditions. Mater. Sci. Eng. C 2013, 33, 3935-3941. [CrossRef] [PubMed]

45. Ehrlich, H.; Maldonado, M.; Spindler, K.D.; Eckert, C.; Hanke, T.; Born, R.; Goebel, C.; Simon, P.; Heinemann, S.; Worch, H. First evidence of chitin as a component of the skeletal fibers of marine sponges. Part I. Verongidae (demospongia: Porifera). J. Exp. Zool. 2007, 308B, 347-357. [CrossRef] [PubMed] 
46. Ehrlich, H.; Ilan, M.; Maldonado, M.; Muricy, G.; Bavestrello, G.; Kljajic, Z.; Carballo, J.L.; Schiaparelli, S.; Ereskovsky, A.; Schupp, P.; et al. Three-dimensional chitin-based scaffolds from Verongida sponges (Demospongiae: Porifera). Part I. Isolation and identification of chitin. Int. J. Biol. Macromol. 2010, 47, 141-145. [CrossRef] [PubMed]

47. Jesionowski, T.; Norman, M.; Zółtowska-Aksamitowska, S.; Petrenko, I.; Joseph, Y.; Ehrlich, H. Marine spongin: Naturally prefabricated 3D scaffold-based biomaterial. Mar. Drugs 2018, 16, 88. [CrossRef]

48. Wysokowski, M.; Motylenko, M.; Bazhenov, V.V.; Stawski, D.; Petrenko, I.; Ehrlich, A.; Behm, T.; Kljajic, Z.; Stelling, A.L.; Jesionowski, T.; et al. Poriferan chitin as a template for hydrothermal zirconia deposition. Front. Mater. Sci. 2013, 7, 248-260. [CrossRef]

49. Wysokowski, M.; Szalaty, T.J.; Jesionowski, T.; Motylenko, M.; Rafaja, D.; Koltsov, I.; Stöcker, H.; Bazhenov, V.V.; Ehrlich, H.; Stelling, A.L.; et al. Extreme biomimetic approach for synthesis of nanocrystalline chitin-(Ti,Zr) $\mathrm{O}_{2}$ multiphase composites. Mater. Chem. Phys. 2017, 188, 115-124. [CrossRef]

50. Mutsenko, V.; Gryshkov, O.; Rogulska, O.; Lode, A.; Petrenko, A.Y.; Gelinsky, M.; Glasmache, B.; Ehrlich, H. Chitinous scaffolds from marine sponges for tissue engineering. In Marine-Derived Biomaterials for Tissue Engineering Applications Chitinous; Springer Nature: Singapore, 2019; pp. 285-307.

51. Mutsenko, V.V.; Gryshkov, O.; Lauterboeck, L.; Rogulska, O.; Tarusin, D.N.; Bazhenov, V.V.; Schütz, K.; Brüggemeier, S.; Gossla, E.; Akkineni, A.R.; et al. Novel chitin scaffolds derived from marine sponge Ianthella basta for tissue engineering approaches based on human mesenchymal stromal cells: Biocompatibility and cryopreservation. Int. J. Biol. Macromol. 2017, 104, 1955-1965. [CrossRef] [PubMed]

52. Petrenko, I.; Khrunyk, Y.; Voronkina, A.; Kovalchuk, V.; Fursov, A.; Tsurkan, D.; Ivanenko, V. Poriferan chitin: 3D scaffolds from nano- to macroscale. A review. Lett. Appl. NanoBioScience 2020, 9, 1004-1014.

53. Rogulska, O.Y.; Mutsenko, V.V.; Revenko, E.B.; Petrenko, Y.A.; Ehrlich, H.; Petrenko, A.Y. Culture and differentiation of human adipose tissue mesenchymal stromal cells within carriers based on sea sponge chitin skeletons. Stem Cell Day 2013, 23, 267-270.

54. Schleuter, D.; Günther, A.; Paasch, S.; Ehrlich, H.; Kljajić, Z.; Hanke, T.; Bernhard, G.; Brunner, E. Chitin-based renewable materials from marine sponges for uranium adsorption. Carbohydr. Polym. 2013, 92, 712-718. [CrossRef] [PubMed]

55. Bechmann, N.; Ehrlich, H.; Eisenhofer, G.; Ehrlich, A.; Meschke, S.; Ziegler, C.G.; Bornstein, S.R. Anti-tumorigenic and anti-metastatic activity of the sponge-derived marine drugs aeroplysinin-1 and isofistularin-3 against pheochromocytoma in vitro. Mar. Drugs 2018, 16, 172. [CrossRef] [PubMed]

56. Drechsel, A.; Helm, J.; Ehrlich, H.; Pantovic, S.; Bornstein, S.R.; Bechmann, N. Anti-tumor activity vs. normal cell toxicity: Therapeutic potential of the bromotyrosines aerothionin and homoaerothionin in vitro. Mar. Drugs 2020, 18, e236. [CrossRef] [PubMed]

57. Nowacki, K.; Stępniak, I.; Machalowski, T.; Wysokowski, M.; Petrenko, I.; Schimpf, C.; Rafaja, D.; Ziętek, J.; Pantović, S.; Voronkina, A.; et al. Electrochemical method for isolation of chitinous 3D scaffolds from cultivated Aplysina aerophoba marine demosponge and its biomimetic application. Appl. Phys. A 2020, 126, 368. [CrossRef]

58. Mulongo, G.; Mbabazi, J.; Nnamuyomba, P.; Hak-Chol, S. Water bactericidal properties of nanosilver-polyurethane composites. Nanosci. Nanotechnol. 2011, 1, 40-42. [CrossRef]

59. Suchomel, P.; Kvitek, L.; Panacek, A.; Prucek, R.; Hrbac, J. Comparative study of antimicrobial activity of $\mathrm{AgBr}$ and Ag Nanoparticles (NPs). PLoS ONE 2015, 10, e0119202. [CrossRef] [PubMed]

60. Liu, Z.; Guo, W.; Guoa, C.; Liu, S. Fabrication of AgBr nanomaterials as excellent antibacterial agent. RSC Adv. 2015, 5, 72872-72880. [CrossRef]

61. Padervand, M.; Elahifard, M.R.; Meidanshahi, R.V.; Ghasemi, S.; Haghighi, S.; Gholami, M.R. Investigation of the antibacterial and photocatalytic properties of the zeolitic nanosized AgBr/TiO composites. Mater. Sci. Semicond. Process. 2012, 15, 73-79. [CrossRef]

62. Wysokowski, M.; Piasecki, A.; Bazhenov, V.V.; Paukszta, D.; Born, R.; Schupp, P.; Petrenko, I.; Jesionowski, T. Poriferan chitin as the scaffold for nanosilica deposition under hydrothermal synthesis conditions. J. Chitin Chitosan Sci. 2013, 1, 26-33. [CrossRef]

63. Bazhenov, V.V.; Wysokowski, M.; Petrenko, I.; Stawski, D.; Sapozhnikov, P.; Born, R.; Stelling, A.L.; Kaiser, S.; Jesionowski, T. Preparation of monolithic silica-chitin composite under extreme biomimetic conditions. Int. J. Biol. Macromol. 2015, 76, 33-38. [CrossRef] [PubMed] 
64. Ehrlich, H.; Simon, P.; Motylenko, M.; Wysokowski, M.; Bazhenov, V.V.; Galli, R.; Stelling, A.L.; Stawski, D.; Ilan, M.; Stöcker, H.; et al. Extreme Biomimetics: Formation of zirconium dioxide nanophase using chitinous scaffolds under hydrothermal conditions. J. Mater. Chem. B 2013, 1, 5092-5099. [CrossRef] [PubMed]

65. Wysokowski, M.; Motylenko, M.; Stöcker, H.; Bazhenov, V.V.; Langer, E.; Dobrowolska, A.; Czaczyk, K.; Galli, R.; Stelling, A.L.; Behm, T.; et al. An extreme biomimetic approach: Hydrothermal synthesis of $\beta$-chitin/ZnO nanostructured composites. J. Mater. Chem. B 2013, 1, 6469-6476. [CrossRef] [PubMed]

66. Wysokowski, M.; Motylenko, M.; Walter, J.; Lota, G.; Wojciechowski, J.; Stöcker, H.; Galli, R.; Stelling, A.L.; Himcinschi, C.; Niederschlag, E.; et al. Synthesis of nanostructured chitin-hematite composites under extreme biomimetic conditions. RSC Adv. 2014, 4, 61743-61752. [CrossRef]

67. Lutterotti, L.; Matthies, S.; Wenk, H. MAUD: A friendly Java program for Material Analysis Using Diffraction. Comm. Power Diffr. Newsl. 1999, 21, 1-20.

(C) 2020 by the authors. Licensee MDPI, Basel, Switzerland. This article is an open access article distributed under the terms and conditions of the Creative Commons Attribution (CC BY) license (http://creativecommons.org/licenses/by/4.0/). 